\title{
NOTES
}

\section{Tiling Deficient Rectangles with Trominoes}

\author{
J. MARSHALLASH \\ DePaul University \\ Chicago, IL 60614 \\ mash@math.depaul.edu \\ SOLOMON W. GOLOMB \\ Communication Sciences Institute \\ University of Southern California \\ Los Angeles, CA 90089-2565
}

A tromino (rhymes with domino) is a shape made up of three $1 \times 1$ squares assembled as shown.

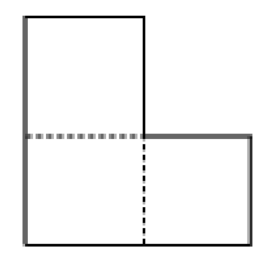

Figure 1 A tromino

We will classify a variety of nearly rectangular shapes into those that can be tiled by trominoes and those that cannot. From now on we will simply say tiled to mean tiled by trominoes. We will consider shapes that are integer-dimensioned rectangles with each dimension at least 2 , and with one or two $1 \times 1$ squares removed. If one square is removed, call the resultant shape a deficient rectangle. If the removed square was a corner square, call the resulting deficient rectangle a dog-eared rectangle. The area of a tromino is 3 , so, evidentially, only shapes whose area is a multiple of 3 can be tiled by trominoes. In this paper we will determine which deficient rectangles with area divisible by 3 are tileable and which are not; in particular, all the dog-eared ones are tileable. We will also get some partial results for the same question for rectangles with two squares removed and remaining area divisible by 3 .

We especially recommend the proof of the Deficient $5 \times 5$ Lemma to the casual reader.

Trominoes were introduced by Golomb [3], who proved that deficient squares whose side length is a power of two can be tiled. Chu and Johnsonbaugh first extended Golomb's work to the general cases of deficient squares [1]. They later went on to rectangles and proved a slightly weaker version [2] of what we call the Deficient Rectangle Theorem. The Proposition in the last section answers a question posed by Chu and Johnsonbaugh [1].

Before proceeding with the business at hand, we will mention a few general facts about trominoes and about another tiling question involving tiles other than trominoes. 
A tromino is a special case of a polyomino, a shape made by connecting certain numbers of $1 \times 1$ squares, each joined together with at least one other square along an edge. The polyomino of area 1 , a single $1 \times 1$ tile is called a monomino. The polyomino of area is two is the domino. Let $P(n)$ be the number of distinct polyominoes of area $n$. For example $P(3)=2$, since there are actually two trominoes, a straight tromino, which has the shape of a $1 \times 3$ rectangle; and the object shown in FIGURE 1 which is called a right tromino when it needs to be distinguished from the straight one, but which will be the only kind of tromino discussed in this paper. Notice that in defining $P$, orientation is ignored. For example, the three objects created by rotating the tromino shown in FIGURE 1 by $90^{\circ}, 180^{\circ}$, and $270^{\circ}$ are not counted as distinct from the original tromino. For results and open questions about the exponentially growing values of $P(n)$, see $[4$, Appendix D].

Four trominoes can be fit together to form a tromino-shaped 4-reptile, that is, a set in the plane that can be tiled by four congruent scaled down copies of itself. A tromino has order 2, which means that the minimum number of trominoes required to form a rectangle is 2, as in FIGURE 2. Finding the order of other polyominoes provides challenging problems $[\mathbf{4}$, Chapter 8]. The entire plane can be tiled in a periodic way by any polyomino of finite order by simply repeating copies of the minimal rectangle.

Roger Penrose has given a remarkably simple aperiodic tiling of the entire plane using copies of only two unit-edged rhombi, one with acute angle $36^{\circ}$ and the other with acute angle $72^{\circ}$ [8]. Tilings are often found in Moorish architecture; some trominoes can be seen in a display case in the Reales Alcazares, a great Arabian style palace built during various epochs in Seville, Spain. A comprehensive and interesting book concerning tiling is Tilings and Patterns [5]. There is lots of information about tiling available on the internet; typing "tromino" into a search engine produced 577 hits. We recommend http://www.ics.uci.edu/ eppstein/junkyard/polyomino. html and http://www . amherst. edu/symbol $\{126\}$ nstarr/.

Elementary results for rectangles A basic tiling result that we will need identifies precisely which rectangles can be tiled. Let's start with some simple cases. First of all, a $2 \times 3$ rectangles can be tiled by trominoes.

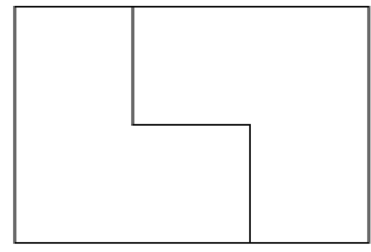

Figure 2 Tiling $R(2,3)$

Denote a rectangle with $i$ rows and $j$ columns by $R(i, j)$. We will indicate decompositions into nonoverlapping subrectangles by means of an additive notation. For example, a $3 i \times 2 j$ rectangle can be decomposed into $i j 3 \times 2$ subrectangles and we write this fact as $R(3 i, 2 j)=\sum_{\mu=1}^{i} \sum_{v=1}^{j} R(3,2)=i j R(3,2)$. It follows from this and the tiling in FIGURE 2 that

$$
\text { any } 3 i \times 2 j \text { or } 2 i \times 3 j \text { rectangle can be tiled. }
$$

From now on, any rectangle decomposed into a combination of $3 i \times 2 j$ subrectangles, $2 i \times 3 j$ subrectangles and trominoes will be considered as successfully tiled by trominoes. Denote the $1 \times 1$ square lying in row $i$ and column $j$ as $(i, j)$. 
Now let's look at some rectangles that cannot be tiled. Suppose that a $3 \times 3$ square $Q$ has been tiled. Some tromino must cover square $(3,1)$. Here are the three possible ways that it can do that.

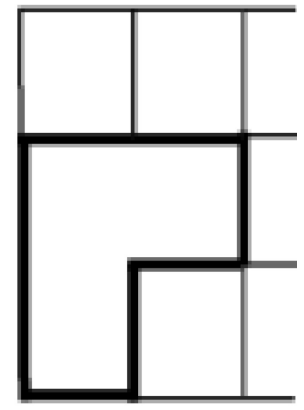

A

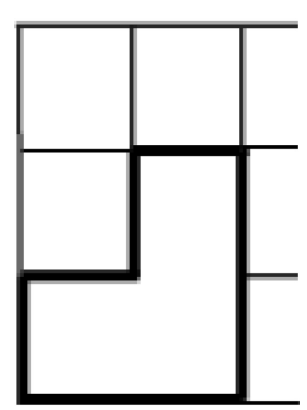

B

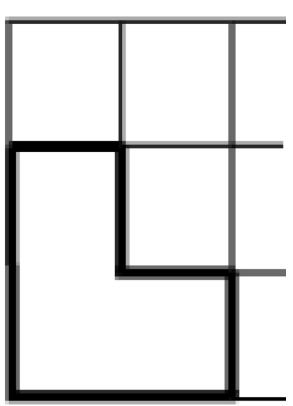

$\mathrm{C}$

Figure 3 An impossibility proof

Orientation $A$ is immediately ruled out, since square $(1,1)$ cannot be tiled. But in cases $B$ and $C$, the tiling must tile the leftmost $3 \times 2$ subrectangle of $Q$, so that the original tiling is also a tiling of the third column of $Q$, which is an $R(3,1)$. This is impossible. Similarly, suppose that a $3 \times 5$ rectangle $R$ has been tiled. This argument shows that the tiling must tile the first two columns of $R$, and hence also the rightmost three columns of $R$. This is a contradiction since we have just shown $3 \times 3$ square to be untileable. Iterating this procedure shows no $R(3$, odd $)$ can be tiled. It turns out that there are no other untileable rectangle with area divisible by 3 .

The integers $m$ and $n$ will always be greater than or equal to 2 .

Chu-Johnsonbaugh TheOREM [2]. An $m \times n$ rectangle can always be tiled by trominoes if 3 divides its area $m n$, except when one dimension is 3 and the other is odd.

The proof of this is not hard. We suggest that the reader give it a try. Here are a few hints. Use fact (1) several times. First do the cases $R(3 k$, even); then do the cases $R(6 k$, odd). This leaves only the cases $R(9+6 k, n)$, where $n \geq 5$ is an odd integer. Reduce such a case to $R(9,5)$. Finally tile $R(9,5)$ by trial and error. If you have trouble with the last step, leaf ahead to the top left picture in FIGURE 9.

Dog-ears An $m \times n$ dog-eared rectangle is an $m \times n$ rectangle with a $1 \times 1$ corner square removed. We will denote the dog-eared rectangle by $R(m, n)^{-}$, so that $R(m, n)^{-}=R(m, n) \backslash\{(1, n)\}$. Note that the area of $R(m, n)^{-}$is $m n-1$. If this rectangle is rotated $180^{\circ}$, a similar figure with missing lower left-hand corner is created. If it is reflected about a central vertical (resp., horizontal) axis, a similar figure with missing upper left-hand (resp., lower right-hand) corner is created. The problem of tiling the original figure is clearly equivalent to tiling any one of the other three, even though the original figure cannot be rotated into either of the last two figures.

Dog-eared Rectangle Theorem. An $m \times n$ dog-eared rectangle can be tiled with trominoes if and only if 3 divides its area.

To understand what this theorem means, note that if $m n$ is congruent to 0 or 2 modulo 3 , then the area of $R(m, n)^{-}$is not congruent to 0 modulo 3 and so that dog-eared rectangle can not be tiled by trominoes, since the area of any region tiled by trominoes must be an integral multiple of 3 . So the only $m \times n$ dog-eared rectangles that 


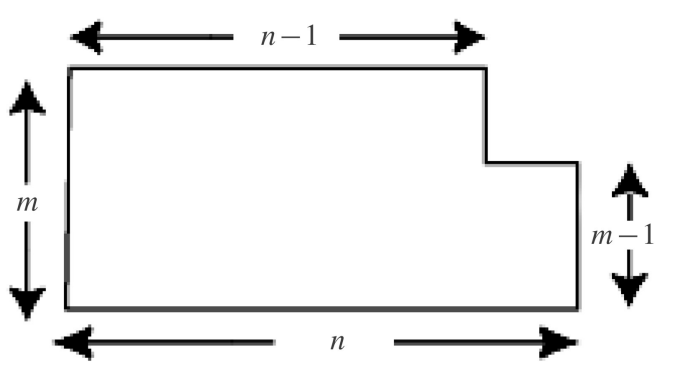

Figure 4 A dog-eared rectangle

could possibly be tiled by trominoes are those for which $m n$ is congruent to 1 . In other words, the only $m \times n$ dog-eared rectangles that could possibly be tiled by trominoes are those for which $m \equiv n \equiv 1(\bmod 3)$ or $m \equiv n \equiv 2(\bmod 3)$, and, indeed, all those dog-eared rectangles can be tiled.

We start with a family of special cases of the Dog-eared Rectangle Theorem, the dog-eared dyadic squares, $R\left(2^{k}, 2^{k}\right)^{-}$. This is a special case of a well-known and beautiful example of mathematical induction [3], [4, page 4], [6, page 45], [9, problem 2.3.38]. If $k=1$, note that $R(2,2)^{-}$is itself a tromino. If $k=2$, see FIGURE 5 for a covering of $R(4,4)^{-}$. In FIGURE $5, R(4,4)^{-}$was tiled by dividing it into 4 quadrants. The upper right quadrant was an $R(2,2)^{-}$while the other three quadrants were all congruent to $R(2,2)$. Then the black tromino covering squares $(2,2),(2,3)$, and $(3,3)$ was placed at the center. This reduced the lower left quadrant to an $R(2,2)^{-}$and the remaining two quadrants to rotations of $R(2,2)^{-}$. In short, the tiling of $R(4,4)^{-}$ was reduced to the tiling of four copies of $R(2,2)^{-}$. The reader should next do $k=3$, by dividing $R(8,8)^{-}$into 4 quadrants, and then covering the central squares $(4,4)$, $(4,5)$, and $(5,5)$ with a tromino. This reduces the tiling of $R(8,8)^{-}$to the tiling of four copies of $R(4,4)^{-}$. The general inductive proof should now be clear.

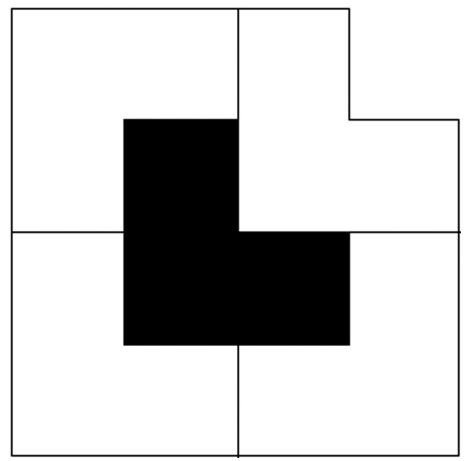

Figure 5 Tiling $R(4,4)^{-}$

Proof of the Dog-eared Rectangle Theorem: Let $m \leq n$. As mentioned above, the necessary condition that 3 divide the area of $R^{-}=R(m, n)^{-}$splits into the cases $m \equiv n \equiv 1(\bmod 3)$ and $m \equiv n \equiv 2(\bmod 3)$. It is not hard to see that the $m \times n$ dogeared rectangle is congruent to the $n \times m$ one. So, in the former case, we have either $R(4,3 k+4)^{-}$with $k \geq 0, R(7,6 k+7)^{-}$with $k \geq 0, R(7,6 k+4)^{-}$with $k \geq 1$, or $R(3 j+4,3 k+4)^{-}$with $j \geq 2$ and $k \geq 2$. There correspond these four decompositions: 


$$
\begin{aligned}
R(4,3 k+4)^{-} & =R(4,3 k)+R(4,4)^{-}, k \geq 0 \\
R(7,6 k+7)^{-} & =R(7,6 k)+R(7,7)^{-}, k \geq 0 \\
R(7,6 k+4)^{-} & =R(7,6 k)+R(4,3)+R(4,4)^{-}, k \geq 1 \text { and } \\
R(3 j+4,3 k+4)^{-} & =R(3 j, 3 k+4)+R(4,3 k)+R(4,4)^{-}, j, k \geq 2 .
\end{aligned}
$$

For the algebraically inclined reader, these decompositions need no further explanation. However, the geometrically inclined reader should draw pictures to visualize them. (All the similar decompositions appearing below have straightforward geometrical interpretations.) Here, in the first three cases, a large rectangle was stripped from the left side of the figure. In the fourth case, first a large rectangle was removed from the bottom, and then another from the left side of what remained. All the full rectangles are tileable by the Chu-Johnsonbaugh Theorem, $R(4,4)^{-}$is tiled as in FIGURE 5 , and the tiling of $R(7,7)^{-}$appears in Chu and Johnsonbaugh [1].

In the latter case, we must tile $R^{-}=R(3 j+2,3 k+2)^{-}$where $0 \leq j \leq k$. If $j \neq 1$, we have

$$
R^{-}=R(3 j, 3 k)+R(3 j, 2)+R(2,3 k)+R(2,2)^{-} .
$$

The first three terms are tiled by the Chu-Johnsonbaugh Theorem, while the last term actually is a tromino. Let $j=1$. Either $k$ is odd, $3 k+2=6 \ell+5$; or else $k$ is even, $3 k+2=6 \ell+8$. Correspondingly, either $R^{-}=R(5,6 \ell+5)^{-}=R(5,6 \ell)+$ $R(5,5)^{-}$where the first term is tiled with the Chu-Johnsonbaugh Theorem and $R(5,5)^{-}$is tiled as in Figure 6, or else $R^{-}=R(5,6 \ell+8)^{-}=R(5,6 \ell)+R(5,8)^{-}$ where again the first term is tiled with the Chu-Johnsonbaugh Theorem and we also have $R(5,8)^{-}=R(5,6)+R(3,2)+R(2,2)^{-}$, the first two terms being tiled by the Chu-Johnsonbaugh Theorem, while the last term is a tromino.

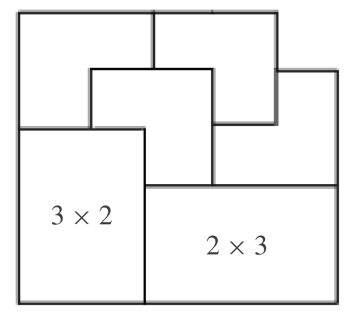

Figure 6 Tiling $R(5,5)^{-}$

Here is an application of the Chu-Johnsonbaugh and Dog-eared Rectangle Theorems. Consider the practical question of tiling as much as possible of any $m \times n$ rectangle, where $m$ and $n$ both exceed 3 . There are 3 cases depending on the value of $m n$ modulo 3. If $m n \equiv 0$, tile the entire rectangle with the Chu-Johnsonbaugh Theorem. If $m n \equiv 1$, remove a single corner square and then use the Dog-eared Rectangle Theorem to tile the rest of the rectangle. If $m n \equiv 2$, we must remove 2 squares. It turns out that if $m n \equiv 2$ and if a corner square and a boundary square adjacent to it are both removed, what remains can always be tiled. This can be proved by methods very similar to those used to prove the other two theorems. We will leave its proof as an exercise.

Deficiency Call a rectangle with one $1 \times 1$ square missing a deficient rectangle. Thus to the rectangle $R(m, n)$ correspond $m n$ deficient rectangles, each being formed by 
removing one square from $R(m, n)$; exactly 4 of these are congruent to $R(m, n)^{-}$. The question of whether a deficient rectangle can be tiled with trominoes is clearly equivalent to the question of whether the full rectangle consisting of the disjoint union of the deficient rectangle and the $1 \times 1$ square can be tiled by a set of trominoes and a single monomino, with the monomino covering the missing square.

Say that a $1 \times 1$ square is good if its removal from a full $m \times n$ rectangle produces a deficient rectangle that can be tiled. We will now enumerate some $m \times n$ deficient rectangles that cannot be tiled, even though 3 divides $m n-1$. This enumeration will be very precise in the sense that for each $m$ and $n$ the location of the bad squares will be specified. In the very interesting case when $m=n=5$, the following lemma produces 16 bad squares.

DEFICIENT $5 \times 5$ LEMMA. If the square $(i, j)$ is removed from the $5 \times 5$ rectangles where either $i$ or $j$ is even, then the resulting shape is not tileable.

Proof. Form a kind of checkerboard design by marking each of the nine squares

$$
\left\{\begin{array}{lll}
(1,1), & (1,3), & (1,5), \\
(3,1), & (3,3), & (3,5), \\
(5,1), & (5,3), & (5,5),
\end{array}\right\}
$$

and assume that one of the 16 unmarked squares has been removed from $R(5,5)$ to form $R^{-}$. Then a proposed tiling of $R^{-}$must contain one tromino for each of the 9 marked squares, so that tiling must have area at least $9 \cdot 3=27$, which is absurd since the area of $R^{-}$is 24 . Thus all 16 of the unmarked squares are bad.

Next we note that bad squares can also occur when $(m, n)=(2,5+3 k), k=$ $0,1,2, \ldots$ Here some bad squares are those of the form $(x, 3 j), j=1,2, \ldots, k+1$, $x=1$ or 2 . By symmetry we may assume that $x=1$. To show that $R(2,5+3 k) \backslash$ $\{(1,3 j)\}$ cannot be tiled, assume the opposite and let $T$ be the tromino covering the square $(2,3 j)$. Then to the left of $T+\{(1,3 j)\}$ lies either the rectangle $R(2,3 j-1)$ or the rectangle $R(2,3 j-2)$, neither of which has area divisible by 3 .

Finally, the square $(3,2)$ is bad in the $5 \times(5+3 k)$ case, that is, $R(5, n) \backslash\{(3,2)\}$ cannot be tiled. For if $(3,2)$ were good, some tromino $T$ would have to cover the square $(3,1)$. If $T$ lay above $(3,2)$ the square $(1,1)$ could not be reached, otherwise the square $(5,1)$ could not be reached. Symmetrically, $(3,4+3 k)$ is also bad in this case.

Deficient Rectangle Theorem (COMPare [2]). An $m \times n$ deficient rectangle, $2 \leq m \leq n, 3 \mid m n-1$, has a tiling, regardless of the position of the missing square, if and only if (a) neither side has length 2 unless both of them do, and (b) $m \neq 5$. Furthermore, in all the exceptional cases the only bad squares are those enumerated in the preceding discussion.

Proof. For this proof only, we change notation slightly and let $R(m, n)^{-}$denote any $m \times n$ rectangle of deficiency 1 . The "outlier" $R(2,2)^{-}$is tiled with one tromino. First assume that $m \geq 4, m \neq 5$, and $3 \nmid m$. The method of proof is to proceed inductively after treating the cases $m=4,7,8,10$, and 11 individually. If $m \geq 13$, then $m-6>6$ so that we may slice a full rectangle of height 6 off of either the top or the bottom of $R(m, n)^{-}$, that is, $R(m, n)^{-}=R(m-6, n)^{-}+R(6, n)$. Since the last term is tileable by the Chu-Johnsonbaugh Theorem, this first reduces the cases $m \in[13,17]$ to the cases $m \in[7,11]$, then the cases $m \in[19,23]$ to the cases $m \in[13,17]$, and so on.

If $m=4$, write $R(4,3 k+1)^{-}=(k-1) R(4,3)+R(4,4)^{-}$. Apply the ChuJohnsonbaugh Theorem to the first $k-1$ terms. For the last term, observe that in [3], Golomb showed that all $2^{k} \times 2^{k}$ deficient squares can be tiled. (Its proof is an induc- 
tion argument almost identical to the one used above to tile the $2^{k} \times 2^{k}$ dog-eared squares.) If $m=7$, we may write $R(7, n)^{-}=R(3, n)+R(4, n)^{-}$and thus reduce the $m=7$ case to the $m=4$ case when $n$ is even; while if $n$ is odd, 6 divides $n-7$ and $R(7, n)^{-}=((n-7) / 6) R(7,6)+R(7,7)^{-}$is tiled using the Chu-Johnsonbaugh Theorem and reference [2]. If $m=10$; then $R(10, n)^{-}=R(7, n)^{-}+R(3, n)$ so that the Chu-Johnsonbaugh Theorem provides a reduction to the $m=7$ case if $n$ is even, while $R(10, n)^{-}=R(10, n-3)^{-}+R(10,3)$ reduces the odd $n$ case to the even $n$ case. If $m=8, R(8, n)^{-}=R(8,8+3 k)^{-}=k R(8,3)+R(8,8)^{-}$is tiled by the ChuJohnsonbaugh Theorem and reference [3]. Finally if $m=11, n$ must be congruent to either 8 or 11 modulo 6 . If $n=8+6 k, R(11,8+6 k)^{-}=k R(11,6)+R(11,8)^{-}$with the first terms tiled by the Chu-Johnsonbaugh Theorem and the last term tiled by the $m=8$ case since $R(11,8)^{-}=R(8,11)^{-}$, while if $n=11+6 k, R(11,11+6 k)^{-}=$ $k R(11,6)+R(11,11)^{-}$; the first terms are tiled by the Chu-Johnsonbaugh Theorem and the tiling of the last term can be found in reference [1].

In view of the treatment of all the bad cases before the statement of this theorem, it remains only to analyze the exceptional good cases. Since 3 divides $m n-1$, if $m=2$, we must have $n=2+3 k, k=0,1, \ldots$, while if $m=5$, we must have $n=5+3 k, k=0,1,2, \ldots$ Also notice that the $(m, n)=(2,2)$ case is not an exception. We'll start with the $5 \times 5$ good cases. The tiling in FIGURE 6 above shows that $(1,5)$ is good, while these two tilings show $(3,5)$ and $(3,3)$ to be good. Symmetry considerations show that the remaining six marked tiles are also good. Thus all nine marked tiles are good.
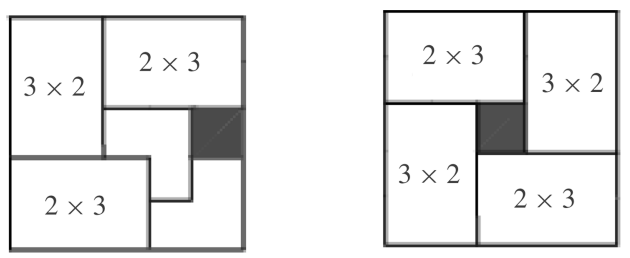

Figure 7 Tiling deficient $5 \times 5$ rectangles

Next, if $(m, n)=(2,5+3 k), k=0,1, \ldots$, we determine to be good all the squares of the form $(x, 3 j+1)$ or $(x, 3 j+2), j=0,1, \ldots, k+1$, where $x=1$ or 2 . In fact, we may write any of these as $(k+1) R(2,3)+R(2,2)^{-}$, apply (1) to each of the first $k+1$ terms, and use one more tromino to cover $R(2,2)^{-}$.

It remains to treat the deficient rectangles $R(5,8+3 k)^{-}$, where $k \geq 0$ and the removed square is neither $(3,2)$ nor $(3,7+3 k)$. Assume that all the cases $R(5,8)^{-}$and $R(5,11)^{-}$have been done and that any square removed from now on is not $(3,2)$. Let the square $(i, j)$ be removed from $R(5,14)$. Symmetry allows the assumption $j \leq 7$. If $(i, j) \neq(3,7)$, then the decomposition of the resulting $R(5,14)^{-}$into an $R(5,8)^{-}$ on the left and an $R(5,6)$ on the right allows a tiling, while $R(5,14) \backslash\{(3,7)\}$ is tiled by decomposing it into an $R(5,6)$ on the left and an $R(5,8)^{-}$on the right. The cases of $R(5, n), n \geq 17$ will be treated inductively. Symmetry allows us to consider only $R(5, n) \backslash\{(i, j)\}$ where $j \leq n / 2<n-8$ and where all but 2 tiles of $R(5, n-6)$ are good. Now decompose into $R(5, n-6)^{-}$on the left and $R(5,6)$ on the right. Since $j \neq(n-6)-1$ the first term may be tiled, while the second is tiled with the ChuJohnsonbaugh Theorem.

The cases $R(5,8)$. By symmetry we may assume $i \geq 3$ and $j \leq 4$. Since $(3,2)$ is bad, we have 11 cases to show good. If $i \geq 4$ and $j \in\{1,2,4\}$, then $(i, j)$ is a good square of $R(2,8)$, so the decomposition of $R(5,8)^{-}$into a full upper rectangle 
$R(3,8)$ and a lower $R(2,8)^{-}$works in all six of these cases. There remain the five cases $(i, j)=(3,1),(5,3),(4,3),(3,3)$, and $(3,4)$. These are done in ad hoc fashion in Figure 8.

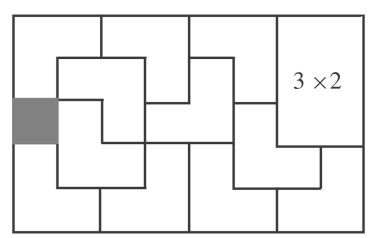

$(3,1)$

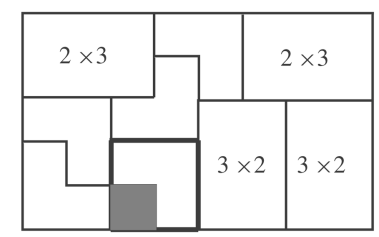

$(5,3)$

Rotate the outlined square $90^{\circ}$ clockwise to tile the $(4,3)$ case.

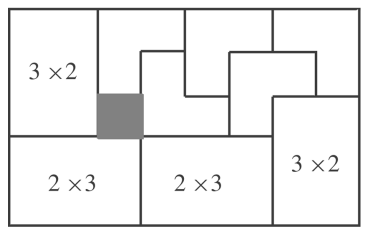

$(3,3)$

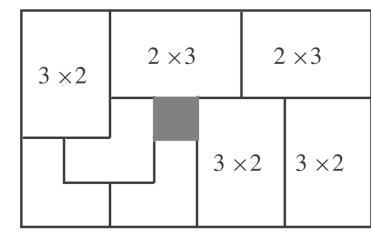

$(3,4)$

Figure 8 Deficient $5 \times 9$ tilings

The cases $R(5,11)$. By symmetry we may assume $i \geq 3$ and $j \leq 6$. Since $(3,2)$ is bad, we have 17 cases to show good. If $i$ and $j$ are both odd, $(i, j)$ is a good square of $R(5,5)$, so the decomposition of $R(5,11)^{-}$into a left $R(5,5)^{-}$and a full right $R(5,6)$ works for these 6 cases. Five of the remaining 11 cases are done in ad hoc fashion in FIGURE 9. A dark outlined $2 \times 2$ square appears in the tiling for the $(4,1)$ case that is shown as the top left picture of FIGURE 9 . Rotate that square $90^{\circ}$ clockwise to produce a tiling for the $(4,2)$ case; then rotate it another $90^{\circ}$ to produce a tiling for the $(5,2)$

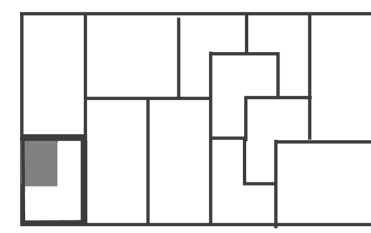

$(4,1)$

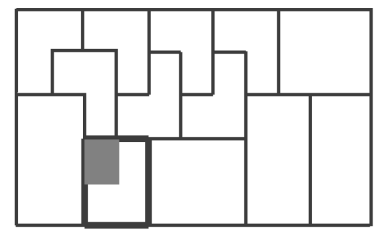

$(4,3)$

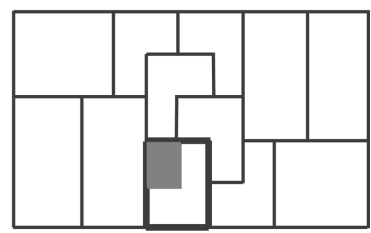

$(4,5)$

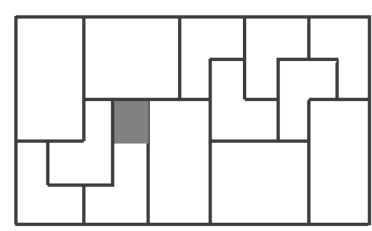

$(3,4)$

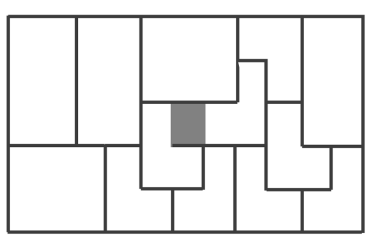

$(3,6)$

Figure 9 Deficient $5 \times 11$ tilings 
case. Similar pairs of rotations produce tilings of the $(4,4)$ and $(5,4)$ cases from the displayed tiling of the $(4,3)$ case, as well as tilings of the $(4,6)$ and $(5,6)$ cases from the displayed tiling of the $(4,5)$ case.

Results and questions about 2-deficiency If two squares are removed from a rectangle, call the resultant shape a 2-deficient rectangle. The following proposition disallows the possibility of making the natural definition of deficiency of order $k, k \geq 2$ and then finding a direct extension of the Deficient Rectangle Theorem for higher deficiencies.

PROPOSITION. No rectangle has the property that no matter which two $1 \times 1$ squares are removed, the remaining shape of area $m n-2$ can be tiled.

For if the squares $(1,2)$ and $(2,1)$ are removed, then the square $(1,1)$ cannot be covered by a tromino. (This also shows the proposition still holds even if "tiling" is extended to mean "tiling by any collection of polyominoes which contains no monomino.")

Even though there will not be a direct analogue of the Deficient Rectangle Theorem, there is room for some interesting work to be done here. Here is a program for what to do about 2-deficiency. We extend the definition of good to 2-deficiency. A pair of squares is good if their removal from a $m \times n$ rectangle leaves a figure that can be tiled.

Problem. For the general case of 2-deficiency, find all bad pairs of squares for all $m \times n$ rectangles where $m n \equiv 2(\bmod 3)$. Slightly less generally, exactly when can such a rectangle be covered by one domino and $(m n-2) / 3$ trominoes?

On the negative side, as we pointed out in the proof of the Proposition, the pair $\{(2,1),(1,2)\}$ is bad, that is, if square $(2,1)$ and square $(1,2)$ are removed, then no tromino can cover square $(1,1)$. On the positive side, recall that in the application given after the proof of the Dog-eared Rectangle Theorem we pointed out that a tiling is always possible if the two removed squares are adjacent and in a corner of the rectangle. In other words, if $m n \equiv 2(\bmod 3)$, then the pair $\{(1, n),(2, n)\}$ is good. Now consider the $5 \times 7$ case. As in the analysis of the $5 \times 5$ case for deficient rectangles done above, form a checkerboard-like pattern by marking each of the 12 squares that have both coordinates odd and assume that two of the 23 unmarked squares have been removed from $R(5,7)$ to form $R^{=}$. Then a proposed tiling of $R^{=}$ must contain one tromino for each of the 12 marked squares, so that tiling must have area at least $12 \cdot 3=36$, which is absurd since the area of $R^{=}$is 33. This reasoning disqualifies $\left(\begin{array}{c}23 \\ 2\end{array}\right)=253$ pairs. Similar reasoning identifies a large number of bad pairs for $R(5,13), \ldots, R(5,7+6 k), \ldots$.

Acknowledgments. Marshall Ash had profitable discussions with Ayse Sahin and Roger Jones. Martin Gardner provided encouragement and some references. Elizabeth Breuer motivated the application given after the proof of the Dog-eared Rectangle Theorem. Jerrold Griggs and a referee provided helpful historical information. We thank the referees for style-improving suggestions. This research was partially supported by NSF grant DMS 9707011 and a grant from the Faculty and Development Program of the College of Liberal Arts and Sciences, DePaul University. 


\section{REFERENCES}

1. I-P. Chu and R. Johnsonbaugh, Tiling deficient boards with trominoes, this MAGAZINE 59 (1986), 34-40, MR 87c:05044.

2. —— Tiling boards with trominoes, J. Rec. Math. 18 (1985-86), 188-193.

3. S. W. Golomb, Checker Boards and Polyominoes, Amer. Math. Monthly, 61 (1954), 675-682.

4. - Polyominoes. Puzzles, patterns, problems, and packings, 2nd ed., Princeton University Press, Princeton, 1994, MR95k:00006.

5. B. Grünbaum and G. C. Shephard, Tilings and Patterns, W. H. Freeman, New York, 1986.

6. R. Johnsonbaugh, Discrete Mathematics, 5th ed., Prentice Hall, Upper Saddle River, NJ, 2001.

7. Amer. Math. Monthly Problem 10641, Proposed by J. R. Griggs, 105 (1998), 175; solution by Michael Woltermann, 107 (2000), 179.

8. R. Penrose, The Emperor's New Mind, Oxford University Press, New York, 1989.

9. P. Zeitz, The Art and Craft of Problem Solving, John Wiley, New York, 1999, MR 2000f:00001. 